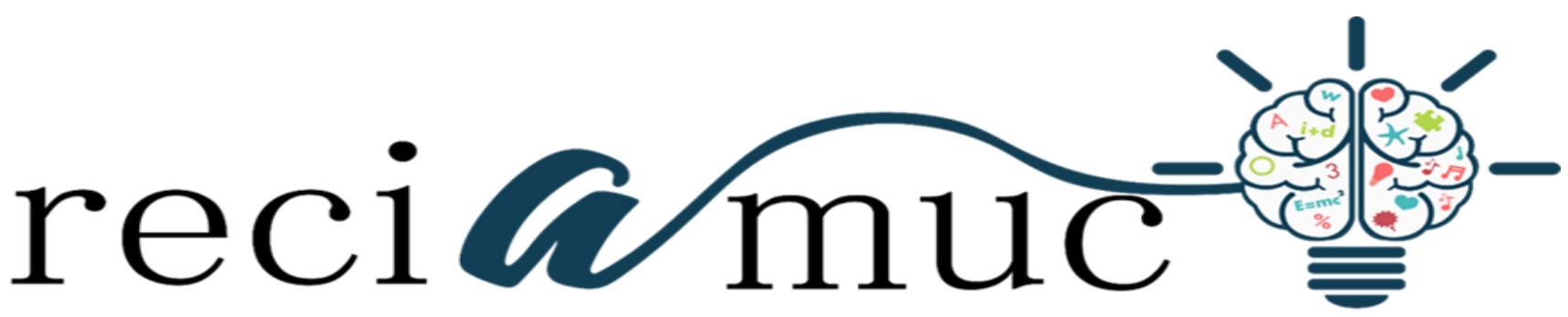

Revista cientifica de investigación actualización del mundo de las ciencias

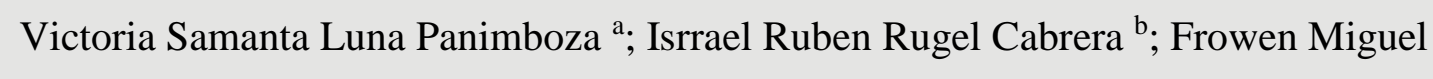
Zambrano Solorzano ${ }^{c}$; Ligia Elena Panezo Carbo ${ }^{d}$

Dolor postquirúrgico por vía epidural y su mejor tratamiento

Post-surgical epidural pain and its best treatment

Revista Científica de Investigación actualización del mundo de las Ciencias. Vol. 3 núm., 2, abril, ISSN: 2588-0748, 2018, pp. 950-975

DOI: 10.26820/reciamuc/3.(2).abril.2019.950-975

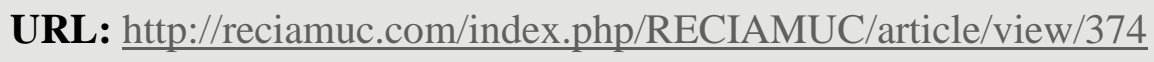

Código UNESCO: 3205 Medicina Interna

Tipo de Investigación: Artículo de Revisión

Editorial Saberes del Conocimiento

Recibido: 21/02/2019

Aceptado: 10/03/2019

Publicado: 30/04/2019

Correspondencia: victoria_luna19@outlook.es
a. Médico; victoria_luna19@outlook.es
b. Médico; dr.isruca@gmail.com
c. Médico;
d. Médico; queen_black85@hotmail.com 


\section{Dolor postquirúrgico por vía epidural y su mejor tratamiento}

Vol. 3, núm. 2., (2019)

Victoria Samanta Luna Panimboza; Isrrael Ruben Rugel Cabrera; Frowen Miguel Zambrano

Solorzano; Ligia Elena Panezo Carbo

\section{RESUMEN}

Actualmente el control del dolor agudo postquirúrgico ha sido un reto para los anestesiólogos y en general para el equipo involucrado durante una cirugía, razón por la cual, las ciencias médicas logran nuevos avances científicos caracterizados por la utilización de métodos avalados, que dan resultados eficientes. Dentro de este orden de ideas, se encuentra la utilización de los analgésicos locales por vía epidural alternativa que busca la reducción del dolor postquirúrgico; la misma no es más que una punción en la espalda a nivel lumbar con una aguja fina y se filtra el anestésico local. Una vez que la piel está anestesiada se introduce una aguja de mayor calibre para colocar un tubo o catéter en el canal raquídeo por fuera de las meninges, de esta forma, se puede administrar el respectivo analgésico cuya función es reducir los efectos del dolor según la dosis administrada en un lapso entre diez a quince minutos. Este nuevo plan postquirúrgico asegura al paciente una recuperación más rápida, reduce temporalmente la inflamación de la zona que produce el dolor y así evita la posibilidad de hacer procedimientos invasivos. Sin embargo, el médico al momento de considerar este tratamiento, debe estimar algunas evaluaciones previas que le garanticen tener resultados viables para el paciente; pues, el mismo está absolutamente contraindicado en caso de infecciones de la piel en la zona de la punción, infección crónica de la columna vertebral, alergias a los anestésicos locales, alteraciones de coagulación, hemorragia intensa o algunas cardiopatías. Argumentos, que permiten la realización de este artículo, cuyo objetivo es analizar el tratamiento del dolor postquirúrgico por vía epidural. De este modo, se puede dar continuidad al proceso investigativo determinado por la revisión bibliográfica debido a su correspondencia con las investigaciones documentales, actividades que dieron oportunidad para elaborar las conclusiones pertinentes.

Palabras Claves: Tratamiento del Dolor Postquirúrgico; Vía Epidural; Tratamiento. 


\title{
Dolor postquirúrgico por vía epidural y su mejor tratamiento
}

Vol. 3, núm. 2., (2019)

Victoria Samanta Luna Panimboza; Isrrael Ruben Rugel Cabrera; Frowen Miguel Zambrano Solorzano; Ligia Elena Panezo Carbo

\begin{abstract}
Currently acute post-surgical pain control has been a challenge for anesthesiologists and in general for the team involved during a surgery, reason why medical science achieved new scientific developments characterized by the use of supported methods, which give efficient results. In this order of ideas, is the use of local analgesics through alternative epidural which seeks the reduction of postoperative pain; it is nothing more than a tap on the back at the lumbar level with a fine needle and local anesthetic is filtered. Once the skin is anesthetized, a larger gauge needle is inserted to place a tube or catheter in the spinal canal outside the meninges, thus administering the respective painkiller whose function is to reduce the effects of pain according to the dose to provide in a span of ten to fifteen minutes. This new post-surgical plan ensures the patient a faster recovery, temporarily reduces inflammation of the area that produces the pain and thus avoids the possibility of invasive procedures. However, the physician at the time of considering this treatment, should estimate some previous assessments that ensure you have viable results for the patient; Therefore it is absolutely contraindicated in case of infections of the skin in the area of the puncture, chronic infection of the spine, allergies to local anesthetics, alterations of coagulation, intense hemorrhage or some heart. Arguments, which allow the implementation of this article, whose objective is to analyze the treatment of postoperative pain through the epidural. In this way, you can give continuity the investigative process determined by the review because of its correspondence with documentary research, activities that gave opportunity for the relevant conclusions.
\end{abstract}

Key Words: Post-surgical Pain Treatment; Epidural way; Treatment. 


\section{Dolor postquirúrgico por vía epidural y su mejor tratamiento}

Vol. 3, núm. 2., (2019)

Victoria Samanta Luna Panimboza; Isrrael Ruben Rugel Cabrera; Frowen Miguel Zambrano Solorzano; Ligia Elena Panezo Carbo

\section{Introducción.}

El dolor ha estado irremisiblemente unido al hombre en todas las épocas, desde su nacimiento hasta su muerte y ha sido sin duda alguna, un importante impulso para el desarrollo de las Ciencias de la Salud. El concepto de dolor y su tratamiento, desde épocas de antiguas civilizaciones, ha estado ligado a la cultura y costumbres mágico-religiosas. Los avances científicos acontecido en los últimos siglos han logrado un mejor entendimiento y manejo del dolor aunque el componente cultural sigue teniendo un gran impacto en la humanidad.

Por lo tanto, el dolor puede considerarse un acompañante innato del hombre desde su nacimiento, el mismo es visto como el más terrible de los señores de la humanidad. Dentro de los diferentes tipos de dolor se encuentra el postquirúrgico, que no es más que una respuesta emitida por el organismo, como resultado a la nocicepción que involucra cuatro procesos fisiológicos, como la transducción que permite a los estímulos nocivos convertirse en actividad eléctrica en las terminaciones sensitivas de los nervios; de esta manera, se logra la respectiva trasmisión o propagación de los impulsos por el sistema nervioso sensitivo para luego dar cabida a la modulación, mediante el cual es modificado el número de influencias nerviosas, finalmente se llega a la percepción que va a tener injerencia con la psiquis del paciente para crear la experiencia emocional subjetiva que permitirá la sensación del respectivo estímulo representado mediante la aparición del dolor.

En relación a estos planteamientos, Torres (2015) indica que "el dolor postquirúrgico es un proceso que aparece como consecuencia a la intervención realizada al paciente". (p.22). Según la conceptualización, se puede indicar que la aparición del dolor postquirúrgico, no es más 


\section{Dolor postquirúrgico por vía epidural y su mejor tratamiento}

Vol. 3, núm. 2., (2019)

Victoria Samanta Luna Panimboza; Isrrael Ruben Rugel Cabrera; Frowen Miguel Zambrano Solorzano; Ligia Elena Panezo Carbo

que el resultado registrado en el paciente como consecuencia de la intervención quirúrgica que la persona es sometida, donde existen múltiples factores asociados a la variabilidad del mismo dolor, para lograr resultados viables, es importante aplicar un tratamiento cónsono con las necesidades del paciente.

Por ello, la aplicación del tratamiento analgésico mediante la vía epidural viene a representar una actuación del anestesiólogo dentro del espacio que se encuentra alrededor de la médula espinal, conocido como el espacio epidural, para proporcionar alivio temporal o prolongado del dolor o la inflamación. Este espacio epidural es la parte más externa del canal vertebral. De esta manera, los esteroides, anestésicos y medicaciones antiinflamatorias habitualmente se administran por inyección epidural que busca simplemente reducir el dolor e hinchazón presentados alrededor de las raíces de los nervios de la columna, y nervios lesionados que con el tiempo quizás se sanen.

En consecuencia, el dolor postquirúrgico se controla con varios medicamentos (analgésicos). El tipo adecuado, administración y dosis de los medicamentos dependen del tipo de cirugía y la recuperación prevista, así como de las propias necesidades del paciente. Para Torres (ob.cit), "la utilización de los opioides lipofílicos producen una analgesia de corta duración (1-4 h), que los hace útiles para el control del dolor post quirúrgico inmediato”. (p.25). Es decir, mediante el tratamiento epidural realizado con la utilización de los lipofilicos, el médico logra ofrecer a los pacientes una reducción inmediata del dolor postquirúrgico, teniendo efectos favorables para su recuperación. 


\section{Dolor postquirúrgico por vía epidural y su mejor tratamiento}

Vol. 3, núm. 2., (2019)

Victoria Samanta Luna Panimboza; Isrrael Ruben Rugel Cabrera; Frowen Miguel Zambrano Solorzano; Ligia Elena Panezo Carbo

De acuerdo con estos aportes, se puede decir que mediante la utilización del tratamiento epidural para el control del dolor postquirúrgico asumido por cualquier opioide depositado en el espacio epidural o intratecal, producirá una analgesia selectiva espinal superior a la conseguida por cualquier otra vía de administración y desprovista de los efectos adversos más temidos como la depresión respiratoria. No obstante, es importante acotar que, una mayor utilización de los opioides condiciona en los pacientes hacia unos cambios fisiopatológicos que les predisponen a una mayor necesidad. Pues, un medicamento administrado vía epidural deberá atravesar además del contenido del propio espacio epidural, las meninges, el líquido cefalorraquídeo y la sustancia blanca.

En razón de los planteamientos anteriores, se puede precisar que la realización de tratamientos mediante el manejo epidural, hace posible la reducción del dolor postquirúrgico, pero los mismos debido a su composición química, llegan a generar efectos secundarios que deben ser considerados por el médico, a fin de recomendar la dosis correspondiente y el tiempo para la misma. Aspectos de significativa importancia en el desarrollo de este artículo, pues, hace posible analizar el tratamiento del dolor postquirúrgico por vía epidural, desde una óptica reflexiva, para llegar a plasmar nuevas ideas capaces de redimensionar los conceptos subyacentes en las características del tema a tratar.

\section{Método.}

El proceso investigativo, para darle correspondencia al propósito previamente establecido, lleva a considerar pertinente la ubicación del método como herramienta esencial para adecuar los contenidos referidos al tratamiento del dolor postquirúrgico por vía epidural y 


\section{Dolor postquirúrgico por vía epidural y su mejor tratamiento}

Vol. 3, núm. 2., (2019)

Victoria Samanta Luna Panimboza; Isrrael Ruben Rugel Cabrera; Frowen Miguel Zambrano Solorzano; Ligia Elena Panezo Carbo

en función de las diferentes apreciaciones se selecciona el método que hace posible redimensionar los eventos encargados de estructurar la recopilación de la información necesaria para la respectiva sustentación del tema seleccionado.

Como método para la investigación se utiliza el análisis e interpretación de datos académicos, información y diferentes conceptos para orientar en forma precisa una posición, así mismo Mata (2018) define al método como: "una estrategia con la que se observa y reflexiona sistemáticamente sobre realidades teóricas y empíricas usando para ello diferentes tipos de documentos donde se indaga, interpreta, presenta datos e información sobre un tema determinado" (p.22). De lo anterior citado, se puede reflexionar que la interpretación de diferentes realidades es necesarias para lograr presentar información orientada en forma correcta y académica, dándole sentido coherente a la investigación.

Por ello, es importante ubicar este artículo en un campo de las ciencias sociales, pues, su relación con un campo metodológico, se convierte en la fuente esencial para encaminar el hecho investigativo, dentro de un campo científico particular, que hace posible responder a las necesidades de indagar. De allí, que se consideró pertinente el método deductivo, dicha selección hizo posible, desglosar los contenidos de forma general, para luego elaborar las apreciaciones que sirvieron de base en la construcción definitiva del artículo.

\section{Tipo de Investigación}

Desde el punto de vista metodológico es una investigación documental, donde se utiliza herramientas para conocer principios y procedimientos que permitan desarrollar la información y el conocimiento. Según Palella y Martins (2010)), la investigación documental "se concreta 


\section{Dolor postquirúrgico por vía epidural y su mejor tratamiento}

Vol. 3, núm. 2., (2019)

Victoria Samanta Luna Panimboza; Isrrael Ruben Rugel Cabrera; Frowen Miguel Zambrano Solorzano; Ligia Elena Panezo Carbo

exclusivamente en la recopilación de información en diversas fuentes. Indaga sobre un tema en documentos escritos”. (p. 99). Es decir, para concretar esta investigación documental, se puede indicar que la misma fue realizada cumpliendo la metodología del método científico, al interpretar y analizar informaciones de documentos académicos vinculados al tema para logra en forma coherente un resultado que apoye y relacione el contenido en función de las necesidades de la investigación.

\section{Fuentes Documentales}

La interpretación de las fuentes informativas de acuerdo a lo requerido en la investigación académica se lleva a incluir los diferentes datos, documentos existentes para generar un orden de ideas adecuadas al tema. Para Angulo (2017) las fuentes documentales "son los documentos, sucesos $\mathrm{u}$ objetos que trasmiten información significativa sobre el origen de la información.”(p.36). De acuerdo con lo citado, se entiende que mediante una adecuada selección de las técnicas documentales, el investigador, logra primero organizar las actividades vinculadas con la lectura rápida, para luego concretar el trabajo bajo una dirección de interpretaciones generales en pro de lograr la respectiva construcción del contenido.

En consecuencia, la realización de este artículo se cumplió mediante la utilización de la lectura e interpretación de documentos relacionados al tema, para generar una matriz nueva de opinión en relación al tema abordado. En otras palabras, se precisaron aquellos aportes teóricos fundamentales para la construcción del cuerpo general de la investigación y en pro de ello, garantizar así de manera eficiente el método previamente seleccionado. 


\section{Dolor postquirúrgico por vía epidural y su mejor tratamiento}

Vol. 3, núm. 2., (2019)

Victoria Samanta Luna Panimboza; Isrrael Ruben Rugel Cabrera; Frowen Miguel Zambrano Solorzano; Ligia Elena Panezo Carbo

\section{Técnicas para la Recolección de la Información}

La recolección de datos en una investigación documental lleva a una estrecha diversidad de técnicas y herramientas para ser utilizadas por el investigador. Logrando centrarse en la lectura de documentos académicos, estadísticas y datos recolectados que permiten realizar un análisis profundo e interpretativo de todos los documentos. Ángulo (ob.cit) la recolección de información "se refiere al uso de una gran diversidad de técnicas y herramientas que pueden ser utilizadas por el analista para desarrollar los sistemas de información” (p.52). Al interpretar lo antes expuesto, se puede decir que el proceso de recolección de información en el desarrollo de la investigación, se cumplió mediante el procesamiento de la temática en forma correcta.

En consecuencia, para el desarrollo de este apartado, se precisó la utilización de la lectura reflexiva, como herramienta que ayuda al investigador a valorar de forma coherente el contenido del trabajo, con el fin de ofrecerlo de manera precisa y cuya pertinencia permita no sólo comprender su contenido, sino dispones de diferentes posibilidades para traspolar sus consideraciones desde diferentes perspectivas.

\section{Resultados.}

En la dinámica científica, la organización de los contenidos a tratar, son una forma ideal para ofrecer desde una perspectiva crítica y reflexiva, las consideraciones que el investigador, agrega al proceso investigativo, por ello, para darle continuidad a las actividades previamente planificadas, se presentan los diferentes eventos caracterizadores del tema. 


\section{Dolor postquirúrgico por vía epidural y su mejor tratamiento}

Vol. 3, núm. 2., (2019)

Victoria Samanta Luna Panimboza; Isrrael Ruben Rugel Cabrera; Frowen Miguel Zambrano Solorzano; Ligia Elena Panezo Carbo

\section{Fisiopatología del Dolor}

El trauma e inflamación que se produce tras el corte y manipulación de los tejidos durante una cirugía, activan nociceptores, que según Mesas (2015) "es convertido en un impulso eléctrico que se trasmite hasta la médula espinal, a través de fibras primarias”. (p.32). Es decir, estas fibras primarias hacen sinapsis con la segunda neurona aferente en el asta dorsal de la médula espinal y se encarga de trasmitir el impulso hasta los centros superiores a través de la vía espinotalámica y la espinorecticular contralateral que son las dos principales vías ascendentes del dolor. Además, hay otras proyecciones que van al córtex cerebral y lleva a la experiencia del dolor.

En consecuencia, al hablar del dolor inflamatorio ocurre debido a la sensibilización del nociceptor que es producida por los mediadores inflamatorios como: citocinas, bradicinina, prostaglandina que se liberan de las células lesionadas en el lugar del daño tisular. La plasticidad de los nociceptores a los mediadores inflamatorios es reversible. La activación del nociceptor baja el umbral produciendo un aumento en la sensibilidad del dolor en el lugar del tejido lesionado. Por ello, el sistema nervioso central demuestra plasticidad como respuesta al dolor y la señal del dolor dentro de la médula espinal puede verse aumentada.

De lo antes expuesto, se desprende que con el aumento de los imputs nociceptivos la relación estímulo - respuesta se altera y entonces se produce un aumento de la excitabilidad de las neuronas, clínicamente esto se manifiesta como una respuesta aumenta a un estímulo doloroso (hiperalgesia) y dolor tras un estímulo táctil no doloroso (alodinia). En consecuencia, el dolor en el paciente quirúrgico se encuentra estrechamente relacionado con la lesión tisular 


\section{Dolor postquirúrgico por vía epidural y su mejor tratamiento}

Vol. 3, núm. 2., (2019)

Victoria Samanta Luna Panimboza; Isrrael Ruben Rugel Cabrera; Frowen Miguel Zambrano Solorzano; Ligia Elena Panezo Carbo

ocasionada por el proceso quirúrgico realizado y junto con otros factores que van a desencadenar respuestas como la inflamatoria metabólica endocrina (eje hipotálamo-hipofisario-suprarrenal) e inmune, dicha respuesta va a tener repercusiones sobre diferentes órganos de los pacientes; debido a que la estimulación de células simpáticas en el hipotálamo y neuronas pre ganglionares en el asta ánterolateral que producirán la liberación de catecolaminas.

Con el fin de ofrecer, una mejor interpretación de los aportes dados anteriormente, se presenta a continuación la figura $\mathrm{n}^{\circ} 1$, mediante la cual, se busca darle al lector una oportunidad de visualizar y comprender la trayectoria que el cuerpo humano posee para responder ante la presencia del dolor postquirúrgico.

\section{Figura $N^{\circ} 1$ Fisiopatología del Dolor}

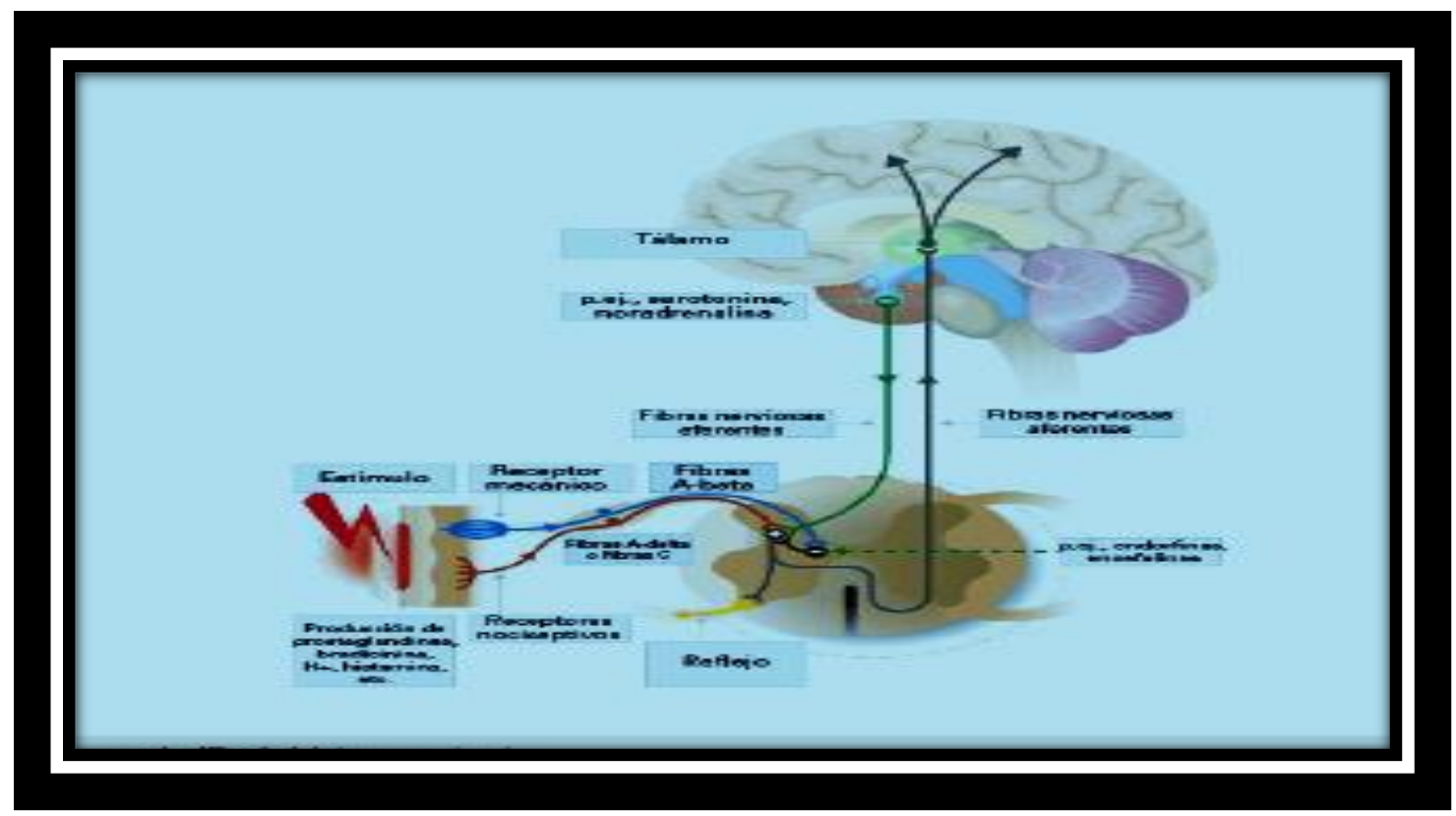

Fuente: Mesas (ob.cit) 


\section{Dolor postquirúrgico por vía epidural y su mejor tratamiento}

Vol. 3, núm. 2., (2019)

Victoria Samanta Luna Panimboza; Isrrael Ruben Rugel Cabrera; Frowen Miguel Zambrano Solorzano; Ligia Elena Panezo Carbo

De acuerdo con la figura anterior, se puede indicar que el dolor es la interacción de mecanismos neurofisiológicos, psicológicos de comportamiento y culturales, siendo que cada paciente presenta su dolor, pues, la nocicepción como proceso, le permite percibir la intensidad del estímulo para así dar una respuesta que refleje un nivel de dolor. Por ello, se ha convertido en el quinto signo vital y es hoy día un tema crítico en la atención del paciente. La mejor definición de dolor es la respaldada por la International Association for the Study of Pain (IASP 2015), "es una experiencia sensorial y emocional desagradable, asociada a una lesión tisular presente o potencial, o descrita en términos de tal lesión”. (p.14)

Dentro de esta perspectiva, el dolor es un complejo fenómeno de respuestas neurofuncionales y subjetivas e individuales a un estímulo, en donde influyen factores emocionales, personalidad, aspectos socioculturales, así como la experiencia previa del paciente al dolor. Se asocia a respuestas autonómicas, psicológicas y conductuales en conjunto provocados por estímulos nocivos a nivel de la piel, estructuras somáticas superficiales y profundas o viscerales, disfunción muscular o visceral.

Según la International Association for the Study of Pain (ob.cit), destaca una clasificación en cuanto al dolor en agudo y crónico, el primero es más reciente, comienza y posee una duración limitada que generalmente tiene una relación causal y temprana con una lesión o enfermedad. Mientras que el dolor crónico persiste a lo largo de períodos más allá del tiempo de cicatrización de la lesión, frecuentemente causado por una causa identificable.

Asimismo, la respectiva organización, precisa la presencia del dolor superficial caracterizado a partir de impulsos de piel, tejidos subcutáneos y mucosas. Otro es el somático 


\section{Dolor postquirúrgico por vía epidural y su mejor tratamiento}

Vol. 3, núm. 2., (2019)

Victoria Samanta Luna Panimboza; Isrrael Ruben Rugel Cabrera; Frowen Miguel Zambrano Solorzano; Ligia Elena Panezo Carbo

profundo, originado en tendones, músculos, articulaciones o huesos. y por último, el visceral causado por una función o enfermedad anormal de un órgano interno o de sus cubiertas, que con frecuencia se acompaña de actividad anormal simpática o parasimpática, asociado a náuseas, vómitos, sudoración y cambios hemodinámicos.

Con el fin de precisar las condiciones del dolor, es importante resaltar que su fisiología y anatomía del dolor como resultado de una intervención quirúrgica, se puede decir que el mismo, se encuentra relacionado con el dolor agudo, que se presenta en el paciente quirúrgico luego del procedimiento. A pesar del avance en técnicas, o fármacos para la disminución del dolor, tanto en países desarrollados como en países en desarrollo, se reportan tasas de dolor postoperatorio que superan el 70\%. En este sentido, Navarro (2018) señala que "la incidencia de dolor moderado a severo en el postoperatorio es entre 8.4 a $47.0 \%$.(p.22).

El manejo adecuado del dolor postoperatorio permitirá una recuperación funcional, permite una de alta de manera más rápida, disminuye el impacto en la calidad de vida, el estado mental, sueño, movilidad y sin las complicaciones derivadas de la activación simpática, respuesta neuroendocrina al estrés, y sus consecuencias cardiovasculares, respiratorias, trombóticas, gastrointestinales, inmunológicas, metabólicas y hematológicas. El manejo adecuado del dolor postoperatorio es un evento costo efectivo en salud, que beneficia al paciente, familiares, e instituciones.

Cabe agregar que el dolor postoperatorio es causado por la estimulación de terminaciones nerviosas libres, las cuales se encuentran en la piel y tejido conectivo, las cuales pueden ser estimuladas por presión, calor, pero más comúnmente por la estimulación química, consecuencia 


\section{Dolor postquirúrgico por vía epidural y su mejor tratamiento}

Vol. 3, núm. 2., (2019)

Victoria Samanta Luna Panimboza; Isrrael Ruben Rugel Cabrera; Frowen Miguel Zambrano Solorzano; Ligia Elena Panezo Carbo

de lesión o inflamación del tejido, misma que resulta de la producción y acumulación de variedad de sustancias como son prostaglandinas, bradicininas, histamina, serotonina, entre otras. Estas sustancias estimulan terminaciones nerviosas libres desencadenándose un impulso nervioso que se transmite a lo largo del nervio periférico hacia el asta dorsal de la médula espinal, donde hace sinapsis con células del tracto espinotalámico que transporta los impulsos a lo largo de la medula a través del tallo cerebral al tálamo de donde son enviados a varias áreas de la corteza cerebral.

Cabe agregar, que la fisiopatología del dolor en los pacientes sometidos a procesos quirúrgicos, muestran claramente que a pesar de los avances farmacológicos y nuevas innovaciones tecnológicas es una evidencia que hoy día los pacientes sometidos a una intervención quirúrgica siguen experimentando dolor postoperatorio. Un control inadecuado del dolor, conlleva una disminución del grado de satisfacción y confort de los pacientes junto con aumento de la morbilidad, estancia hospitalaria y costes asociados. Las complicaciones endocrino-metabólicas se producen por la liberación hormonal inducida por la agresión quirúrgica debido a la estimulación del sistema nervioso autónomo central y periférico.

Es decir, la característica del dolor por efecto quirúrgico, tiene lugar una liberación de hormonas catabólicas (ACTH, ADH, GH, AMPc, cortisol, catecolaminas, renina, angiotensina II, aldosterona y glucagón), una disminución de hormonas anabolizantes (insulina y testosterona) así como una serie de alteraciones metabólicas que incluye hiperglucemia, intolerancia a la glucosa y resistencia a la insulina, catabolismo proteico muscular y lipólisis del tejido adiposo con aumento de los ácidos grasos libres. Como resultado se produce un estado diabetogénico, una negativización del balance nitrogenado, retención hidrosalina, excreción aumentada de 


\section{Dolor postquirúrgico por vía epidural y su mejor tratamiento}

Vol. 3, núm. 2., (2019)

Victoria Samanta Luna Panimboza; Isrrael Ruben Rugel Cabrera; Frowen Miguel Zambrano Solorzano; Ligia Elena Panezo Carbo

potasio, contracción del líquido extracelular, disminución de la capacitancia venosa y aumento de las resistencias periféricas, inotropismo y frecuencia cardiaca.

El deterioro de la función respiratoria es una de las complicaciones más importantes, con una incidencia entre el 5-25\%, elevándose hasta el 65\% cuando se trata de una cirugía torácica o de hemiabdomen superior. Se produce un deterioro respiratorio manifestado como atelectasia, neumonía, derrame, taquipnea, tos, expectoración, fiebre y/o cianosis. Puede producirse el denominado síndrome restrictivo pulmonar postoperatorio donde, a consecuencia del dolor, aparecen espasmos musculares que ocasiona una disminución de la compliance pulmonar y por tanto hipoxemia.

Por ello, la hiperactividad simpática y la liberación de catecolaminas inducidas por el dolor da lugar a taquicardia, aumento de las resistencias periféricas, hipertensión y aumento del consumo de oxigeno miocárdico. Estas complicaciones son más elevadas en aquellos pacientes con factores de riesgo asociado. Los trastornos vasculares periféricos secundarios a la inmovilización del paciente por el dolor, y el vasoespasmo reactivo contribuyen al empeoramiento de la circulación periférica, aumentando el riesgo de trombosis venosa profunda y tromboembolismo. Aún no está claro que un tratamiento adecuado del dolor postoperatorio modifique la morbilidad cardiaca, pero el empleo de analgesia epidural como de morfina a dosis altas pueden mejorar la taquicardia y la isquemia pero no modifican la incidencia de complicaciones más severas.

A consecuencia de la hiperactividad simpática generada tras la agresión quirúrgica, se produce una disminución de la actividad digestiva: disminución de la motilidad gastrointestinal, 


\section{Dolor postquirúrgico por vía epidural y su mejor tratamiento}

Vol. 3, núm. 2., (2019)

Victoria Samanta Luna Panimboza; Isrrael Ruben Rugel Cabrera; Frowen Miguel Zambrano Solorzano; Ligia Elena Panezo Carbo

aumento tono de esfínteres y de las secreciones intestinales acompañado de distensión abdominal, náuseas y vómitos, intolerancia digestiva y reducción del ritmo del peristaltismo normal. Los factores que van a condicional la gravedad de estas complicaciones son la localización de la cirugía, la técnica anestésica y el uso de opioides.

También se pueden producir complicaciones inmunológicas, urológicas y musculo esqueléticas. Se produce leucocitosis, neutropenia, linfopenia, disminución de la quimiotaxis, disminución de la opsonificación, aumento de la capacidad fagocitaria, disminución de la función de los linfocitos B y T, disminución de la secreción de inmunoglobulinas, entre otras alteraciones inmunológicas. Por otro lado, debido al aumento del tono simpático, se produce una inhibición de la motilidad del tracto urinario que conduce a retención urinaria.

Debido a la inmovilidad funcional, consecuencia de la intervención quirúrgica, aparece atrofia muscular que puede ocasionar un retraso en la recuperación. La ansiedad, miedo, angustia, aprensión, depresión son sensaciones unidas al dolor. La ansiedad constituye una de las complicaciones psicológicas más relevantes. Se ha comprobado que pacientes que recibieron técnicas psicológicas preoperatorias experimentaron un menor dolor postoperatorio. Un tratamiento adecuado del dolor postquirúrgico no siempre va ligado a una disminución del estrés emocional del paciente. Un apoyo psicológico, sobre todo en cirugías agresivas o de pronóstico incierto, ayuda a una recuperación más temprana.

\section{Tratamiento por vía epidural para el dolor quirúrgico}

Actualmente a pesar de los avances científicos y tecnológicos en la medicina, ni el dolor agudo ni el crónico suelen recibir un tratamiento adecuado por muy diversas razones, se ha 


\section{Dolor postquirúrgico por vía epidural y su mejor tratamiento}

Vol. 3, núm. 2., (2019)

Victoria Samanta Luna Panimboza; Isrrael Ruben Rugel Cabrera; Frowen Miguel Zambrano Solorzano; Ligia Elena Panezo Carbo

encontrado que hasta el $65 \%$ de los pacientes en el periodo postoperatorio reciben un mal manejo analgésico, esto es debido a que los esquemas analgésicos establecidos no logran alcanzar niveles plasmáticos adecuados o que estos solo se circunscriben a AINE, lo que con lleva a una mala evolución del paciente y aumento de la morbimortalidad postoperatoria. Es por ello que haciendo uso de la vía peridural, permite la administración continua de medicamentos, en estudio como es el caso de fentanil a dos dosis 100 y 200mcg, en combinación con Ropivacaína, evita los valles o picos, y por lo tanto se mantiene una analgesia continua, reflejándose en una mayor satisfacción del paciente.

En vista que el dolor generado por la cirugía es muy difícil de controlar, llegando hasta en ocasiones a un $70 \%$ de falla, esto es debido a las subdosis de analgésicos que se aplican y a las vías de administración. El advenimiento de las bombas de infusión continua tipo PCA y el uso a través del espacio epidural han tenido éxito. Gracias a los resultados obtenidos con las bombas de infusión continua y al desarrollo de nuevos fármacos (opioides, anestésicos locales), se ha logrado que el manejo del dolor tenga un mayor porcentaje de éxito y esto se ve reflejado en un mayor bienestar de los pacientes.

Lo anterior puede traducirse en una evolución postquirúrgica placentera, una recuperación rápida, un menor tiempo intrahospitalario, además de permitir la reincorporación temprana a las actividades cotidianas. Es allí, donde la PCA (Analgesia Controlada por el Paciente), por sus siglas en inglés, originalmente fue descrita en pacientes de parto en la década de los años sesenta, pero esta técnica fue ampliamente reconocida después de su introducción en el campo del dolor postoperatorio a mediados de los ochenta. 


\section{Dolor postquirúrgico por vía epidural y su mejor tratamiento}

Vol. 3, núm. 2., (2019)

Victoria Samanta Luna Panimboza; Isrrael Ruben Rugel Cabrera; Frowen Miguel Zambrano Solorzano; Ligia Elena Panezo Carbo

Debe señalarse que las primeras bombas de PCA surgen por la necesidad de adaptar varios tipos de analgesia y las diversas rutas de administración a las necesidades individuales de cada paciente; estas bombas permitían a las pacientes ajustar la velocidad de flujo de las diluciones analgésicas y posteriormente se desarrollaron sistemas controlados electrónicamente y supervisadas por microprocesadores. Algunos de estos nuevos modelos proporcionan dosis de infusión calculadas a velocidad fija durante un período de tiempo; algunas otras cuentan con un sistema que permite la infusión de una cantidad pre-programada de analgésico, la cual puede ser activada por el paciente (dosis demanda).

La alteración no autorizada de los parámetros de dosificación es prevenida por diversos factores de seguridad que evitan la sobredosificación. El empleo de las bombas PCA aprovechando la vía peridural para el manejo del dolor, ha proporcionado buenos resultados dentro de las primeras 24 horas, las cuales se consideran como el lapso donde ocurre el dolor de más difícil control. Con respecto al dolor, desde el punto de vista clínico se clasifica en agudo y crónico, el primero se caracteriza por conocer su etiología, la cual está condicionada por un daño tisular y la respuesta inflamatoria, presentando una duración aproximada de 24 a 36 h, acompañada de una hiperactividad simpática, manifestada por taquicardia, hipertensión arterial, palidez, sudoración, dilatación pupilar y angustia.

En este particular, se han realizado diversos estudios tanto en niños como en adultos con diferentes esquemas de ropivacaína más fentanilo para su administración en infusión continua en concentraciones desde $0.1 \%$ hasta $20 \mathrm{mg} / \mathrm{h}$ de ropivacaína, obteniéndose una analgésica satisfactoria, siendo que las dosis terapéuticas para la ropivacaína son de 12 a $28 \mathrm{mg} / \mathrm{h}$, y de 6 hasta $14 \mathrm{ml} / \mathrm{h}$ al $2 \%$ para manejo de la analgesia; siendo diferentes las dosis anestésicas que se 


\section{Dolor postquirúrgico por vía epidural y su mejor tratamiento}

Vol. 3, núm. 2., (2019)

Victoria Samanta Luna Panimboza; Isrrael Ruben Rugel Cabrera; Frowen Miguel Zambrano Solorzano; Ligia Elena Panezo Carbo

manejan con concentraciones al $7.5 \%$ con dosis de 113 a $150 \mathrm{mg}$ y volumen de 15 a $20 \mathrm{ml}$. Y el citrato de fentanilo a dosis para analgesia de 1 a $4 \mu \mathrm{g} / \mathrm{ml}$.

En este orden de ideas, se puede indicar que los efectos secundarios que se reportan con respecto a la ropivacaína son: hipotensión, náusea, bradicardia, vómito, cefalea, fiebre, retención urinaria, parestesia, prurito, y para el fentanilo: depresión respiratoria, apnea, rigidez muscular, movimientos mioclónicos, bradicardia, hipotensión, náusea, vómito, mareo, prurito, anafilaxia, todos estos efectos secundarios se pueden presentar. Afortunadamente su incidencia es baja y el porcentaje va a variar de acuerdo a la velocidad de aplicación y la vía de acceso.

El termino umbral al dolor se utiliza para describir una sensibilidad general al dolor en relación a la variabilidad entre distintos individuos, ya que uno puede tener un umbral elevado en relación a los otros, esto implica una diferencia en el sistema nervioso consistente en que esa persona necesita un estímulo mayor para sentir el mismo nivel de dolor que una persona con un umbral normal; aunque también puede representar un sistema nervioso insensible. Entonces el umbral del dolor se define como el estímulo mínimo capaz de evocar dolor con fiabilidad. Al respecto, Navarro (ob.cit), precisa que para estimar el umbral del dolor es importante utilizar la escala verbal numérica (EVN) que se presenta a continuación. 


\section{Dolor postquirúrgico por vía epidural y su mejor tratamiento}

Vol. 3, núm. 2., (2019)

Victoria Samanta Luna Panimboza; Isrrael Ruben Rugel Cabrera; Frowen Miguel Zambrano Solorzano; Ligia Elena Panezo Carbo

\section{Figura $N^{\circ} 2$ Escala Verbal Numérica}

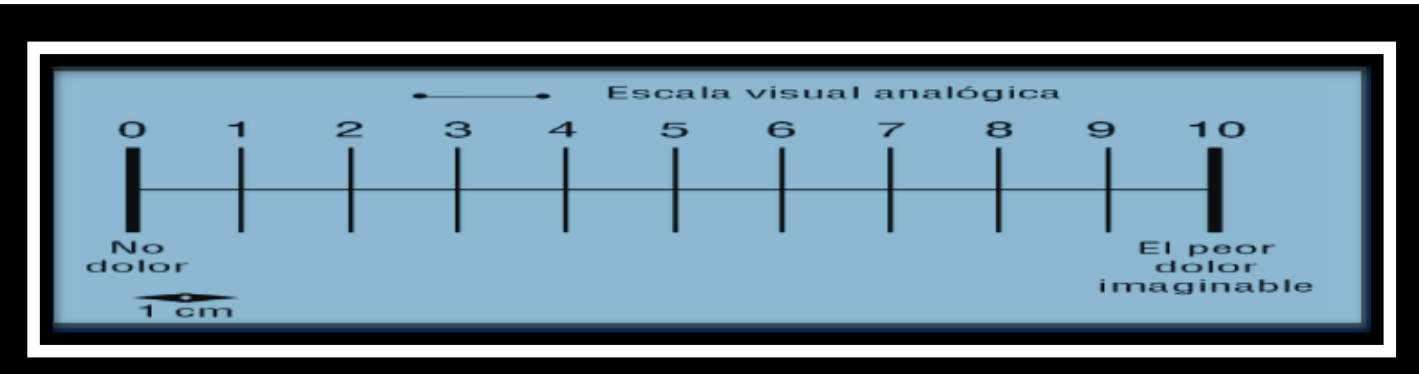

Fuente: Navarro (ob.cit)

Esto lleva a conseguir la medición del dolor es un objetivo difícil ya que es subjetivo y supone los sesgos de la comunicación verbal. En tanto la tolerancia al dolor se define como la capacidad de soportar un estímulo continuó o la máxima intensidad de estímulo tolerada. El dolor es una experiencia personal y subjetiva que comprende una serie de dimensiones: sensitiva -discriminativa, afectiva - emocional y cognitiva - evaluativa; estas tres dimensiones del dolor interactúan unas con otras para proporcionar la información perceptiva sobre la localización, la magnitud y características espaciotemporales de los estímulos nociceptivos, además dan la motivación para la fuga o el ataque y en base a experiencias la probabilidad de éxito al hacer frente al dolor.

Según la literatura se encuentra que la misma apoya el uso de los opioides a través de un catéter para alcanzar una adecuada analgésica postquirúrgica, su uso ha demostrado adecuados niveles de analgesia postquirúrgica; pero se ha observado el prurito como síntoma de mayor frecuencia desagradable que afecta la satisfacción del paciente, una ventaja del uso de la morfina espinal es que por esta vía dura 24 horas. Otro tratamiento importante son los analgésicos 


\section{Dolor postquirúrgico por vía epidural y su mejor tratamiento}

Vol. 3, núm. 2., (2019)

Victoria Samanta Luna Panimboza; Isrrael Ruben Rugel Cabrera; Frowen Miguel Zambrano Solorzano; Ligia Elena Panezo Carbo

opioides son adicionados a los AINE/paracetamol cuando no se obtiene suficiente alivio con estos. Su utilización en dolor agudo postoperatorio y dolor por cáncer está bien fundamentada.

En el campo de la medicina, la introducción de la analgesia controlada por el paciente (PCA) ha permitido el ajuste de dosis de opioides dentro de un amplio abanico de acontecimientos postoperatorios sumado a la disminución de efectos adversos. Se clasifican en: Agonistas puros: se comportan como agonistas y, a veces, selectivos sobre receptores $\mu$. Pertenecen a este grupo: morfina, heroína, petidina, metadona, codeína, dihidrocodeína, hidromorfina, oxicodona, fentanilo y sufentanilo.

Los agonistas-antagonistas mixtos: son capaces de actuar sobre más de un tipo de receptor opioide, $\mu \mathrm{o} \kappa$ comportándose como agonistas sobre el receptor $\kappa$ y como agonistas parciales o antagonistas sobre el $\mu$. Los principales fármacos de este grupo son: pentazocina, ciclazocina, ketociclazocina y butorfanol (no uso en humanos, uso en veterinaria); más discutibles son la nalorgina y la nalbufina. Para algunos autores también deberían incluirse la buprenorfina.

Agonistas parciales: poseen actividad intrínseca sobre receptor $\mu$ inferior a la de los agonistas puros, por lo que en presencia de los mismos podrían comportarse como antagonistas. El más representativo de este grupo es la buprenorfina. Están indicados en el tratamiento del dolor moderado-severo. Dentro de los efectos secundarios más importantes son depresión del sistema nervioso central (depresión respiratoria, supresión reflejo de la tos, somnolencia), vómitos, miosis, exacerbaciones de algunos reflejos espinales u raramente convulsiones. Existe tolerancia a los efectos depresores pero no al estreñimiento ni a la miosis. En el caso de 


\section{Dolor postquirúrgico por vía epidural y su mejor tratamiento}

Vol. 3, núm. 2., (2019)

Victoria Samanta Luna Panimboza; Isrrael Ruben Rugel Cabrera; Frowen Miguel Zambrano Solorzano; Ligia Elena Panezo Carbo

postoperatorio de cirugía abdominal hay que evitar su uso hasta que el ritmo intestinal sea normal por el riesgo de íleo.

La mayoría de los agonistas, agonistas-antagonistas mixtos y agonistas parciales son considerados opiáceos mayores por aliviar o suprimir dolores agudos de gran intensidad, como el dolor postoperatorio, así como dolores crónicos. El techo antiálgico no es igual para todos los opioides, alcanzando mayor eficacia en dolores intensos con los agonistas puros puesto que permiten dosis mayores al no ocasionar reacciones disfóricas y psicomiméticas

No obstante, lo que limita mucho el uso de los opioides en dolor crónico son dos cosas: Sus efectos adversos y el estigma que tiene sobre mal uso y adicción. Los efectos adversos más frecuentes son nausea y vómito, mareo, sedación, constipación, hipogonadismo y depresión respiratoria. La náusea y el vómito suelen auto limitarse al cabo de una semana de tratamiento ya que se desarrolla tolerancia a este efecto con rapidez, pero en lo que ocurre es imperativo administrar un antiemético eficaz. Algo parecido ocurre con mareo y sedación, siendo dosis dependientes. Con la constipación no se presenta tolerancia, por lo que el paciente debe mantenerse con manejo constante para estreñimiento, que va desde abundantes líquidos orales y fibra hasta.

Dentro de este grupo de tratamiento, también se encuentran la analgésica continua epidural (CEA), que según Ruiz (2018) requiere más planificación y trabajo de equipo que la PCA. Aunque la CEA ofrece beneficios bien documentados en pacientes seleccionados, algunos cirujanos pueden tener sus propias opiniones al respecto, de manera que se debería discutir el tratamiento con ellos. De manera ideal, la epidural debe instaurarse antes de la cirugía, por la 


\section{Dolor postquirúrgico por vía epidural y su mejor tratamiento}

Vol. 3, núm. 2., (2019)

Victoria Samanta Luna Panimboza; Isrrael Ruben Rugel Cabrera; Frowen Miguel Zambrano Solorzano; Ligia Elena Panezo Carbo

Unidad de Dolor Agudo o por el anestesiólogo responsable, para proporcionar analgesia intra y postoperatoria. Se utiliza correctamente, el paciente debería llegar a la Unidad de Reanimación Post Anestesia libre de dolor y así permanecer mientras tenga el catéter. No es necesario recordar que puede haber muchas dificultades durante su uso. Los siguientes aspectos pueden ayudar a decidir si la CEA es una buena elección:

Máximo beneficio: toracotomía, cirugía abdominal alta, enfermedad pulmonar previa, obesidad, cirugía reconstructiva de miembros inferiores, sobre todo prótesis de rodilla, que precise equipo de movilización pasiva continúa.

Beneficio modesto: cirugía abdominal baja, cirugía génito -urinaria, ginecología, otros tipos de cirugía ortopédica, y cualquier tipo de cirugía realizada bajo anestesia epidural cuando se prevea la presencia de dolor y el paciente esté ingresado el tiempo suficiente.

Ventajas demostradas de la CEA (en pacientes seleccionados): disminución de la mortalidad peri-operatoria, menor incidencia general de complicaciones, menor tasa de infecciones pulmonares, menor tiempo necesario de intubación y menores costes hospitalarios en pacientes de alto riesgo; mejor analgesia, menor sedación y movilización más precoz en la mayoría de los pacientes.

Contraindicaciones relativas de la CEA: retraso mental o falta de colaboración, bajo nivel de conciencia, problemas lingüísticos e historia de toxicomanía con opiáceos. Estos últimos pueden experimentar un buen alivio del dolor con la CEA, pero pueden precisar mórficos parenterales o epidurales para prevenir un síndrome de abstinencia. 


\section{Dolor postquirúrgico por vía epidural y su mejor tratamiento}

Vol. 3, núm. 2., (2019)

Victoria Samanta Luna Panimboza; Isrrael Ruben Rugel Cabrera; Frowen Miguel Zambrano

Solorzano; Ligia Elena Panezo Carbo

Contraindicaciones absolutas para la CEA: rechazo del paciente, anticoagulación completa, infección local o sepsis. Existen recomendaciones, basadas en la revisión de la literatura, acerca del uso creciente de las heparinas de bajo peso molecular para la profilaxis de la trombosis venosa profunda, especialmente en los pacientes de cirugía ortopédica.

\section{Conclusiones.}

En razón de las consideraciones descritas anteriormente, permite destacar a continuación un grupo reflexivo de interpretaciones que resultan de interés para el lector, entre las cuales se encuentran las siguientes:

La medicina, en un porcentaje elevado de casos, se refiere al tratamiento de las manifestaciones generadas por una lesión o por una enfermedad. El síntoma que mayor frecuencia enfrenta el médico es el dolor; sensación desagradable que experimentan inevitablemente casi todos los seres humanaos y la principal razón de que consuman medicamentos. Ante la presencia de dolor el médico es requerido para su alivio y para eliminar las causas que lo generan. En esta situación el dolor asume el carácter de enfermedad y la eficacia de su tratamiento depende de la comprensión de los elementos anatómicos, fisiológicos, bioquímicos y psicológicos subyacentes.

El manejo del dolor es un problema típicamente interdisciplinario, que va desde el empleo de medicamentos de fácil administración hasta procedimientos neuroquirúrgicos muy complicados. Afortunadamente, en la mayoría de los casos se le puede controlar con fármacos capaces de inducir un estado de analgesia; es decir, insensibilidad o disminución de la sensación 


\section{Dolor postquirúrgico por vía epidural y su mejor tratamiento}

Vol. 3, núm. 2., (2019)

Victoria Samanta Luna Panimboza; Isrrael Ruben Rugel Cabrera; Frowen Miguel Zambrano Solorzano; Ligia Elena Panezo Carbo

del dolor. De hecho, existe un número de medicamentos que, a través de mecanismos diversos, alivian el dolor postquirúrgico.

El dolor postquirúrgico agudo es una reacción fisiológica compleja, por distensión visceral, enfermedad o trauma. Se trata de una manifestación de las reacciones autonómicas psicológicas y conductuales que resultan de una experiencia molesta, sensorial y emocional indeseada. Es uno de los problemas más importantes a los que se enfrentan los pacientes sometidos a un proceso quirúrgico, el dolor afecta a los sistemas cardiovascular, respiratorio y endocrino, un buen manejo analgésico en el postoperatorio puede ayudar a prevenir la presentación de múltiples complicaciones derivadas de las alteraciones de los sistemas mencionados.

El manejo del dolor postquirúrgico sigue siendo difícil y controversial, la utilización de la vía peridural da una mejor alternativa por que ofrece una potencia analgésica superior y porque ayuda a disminuir la morbimortalidad, en bajas concentraciones atenuando las respuesta neuroendocrina al trauma quirúrgico, además de que permite una mejor ventilación pulmonar. Los medicamentos han demostrado ser seguros y eficaces para ser aplicados por esta vía son los anestésicos locales, opioides y los alfa 2 agonistas, el uso combinado a concentraciones bajas como son fentanil 100 y $200 \mathrm{mcg}$ mas Ropivacaína, se obtiene un efecto sinergista y reducen los efectos adversos, lo que permite un acortamiento en el tiempo de estancia hospitalaria, mejorando los costos e impacto favorable en la economía de los pacientes.

A pesar de los avances tecnológicos tanto en equipo biomédico (bombas de infusión continua) como farmacológicos (anestésicos y opioides), el manejo del dolor sigue y seguirá 


\section{Dolor postquirúrgico por vía epidural y su mejor tratamiento}

Vol. 3, núm. 2., (2019)

Victoria Samanta Luna Panimboza; Isrrael Ruben Rugel Cabrera; Frowen Miguel Zambrano Solorzano; Ligia Elena Panezo Carbo

siendo un reto terapéutico, ya que la prevalencia del dolor postquirúrgico continúa siendo elevada debido a la falta de aprovechamiento y/o ignorancia de los avances tecnológicos.

\section{Bibliografía.}

Fernández R, C., \& Gómez L, M. (2018). Dolor agudo y postoperatorio. Colombia: Asociación Colombiana para el Estudio del Dolor.

Mata, O. (2018). Investigación Cientifica. México: Continental.

Mesas, A. (2015). Control del Dolor Postoperatorio. Madrid: Elsevier.

Pain, I. A. (5 de Abril de 2015). https://www.usp-pain.org. Obtenido de https://www.usppain.org

Palella, M. Y. (2010). Metodología de la Investigación Cuantitativa. Caracas: FEDUPEL.

Ruiz, M. (2018). Atención al Paciente Postoperatorio . Actualidad Médica , 36-44.

Torres, A. (2015). Dolor Agudo . Salud Pública, 20-32. 